\title{
Insulin resistance study in diabetes mellitus type II and its correlation with obesity
}

\author{
Saima Usman ${ }^{1, ~ *, ~ S a g h i r ~ A h m a d ~ J a f r i ~}{ }^{2}$ \\ ${ }^{1}$ Dept. of Biology, Brock University, St Catharines, Canada \\ ${ }^{2}$ Fatima Memorial Postgraduate Institute, Lahore, Pakistan \\ Email addresses: \\ dr.saimausman@gmail.com (S. Usman), jafri43@yahoo.com (S. A. Jafri)
}

\section{To cite this article:}

Saima Usman, Saghir Ahmad Jafri. Insulin Resistance Study in Diabetes Mellitus Type II and Its Correlation with Obesity. American Journal of Life Sciences. Vol. 2, No. 2, 2014, pp. 96-102. doi: 10.11648/j.ajls.20140202.20

\begin{abstract}
Background: The earliest detectable abnormality in diabetes mellitus type II (DM II) is the impairment of body's ability to respond to insulin, necessitating extra insulin secretion in an attempt to stimulate the desensitized cells. Obesity is considered as the single most important, reversible, causative environmental factor for insulin resistance (IR) aggravating the metabolic disturbance in DM II. Objectives: We aimed the study to evaluate the association of IR with DM II (its major outcome) and obesity (its main causative factor) in Pakistani diaspora. We measured IR in insulin resistance syndrome (IRS), DM II and obesity by using parameters namely fasting insulin level (FIL), homeostatic model assessment for insulin resistance (HOMA-IR), and quick insulin sensitivity check index (QUICKI), and evaluated their credibility. Materials and Methods: The study included 156 subjects (76 males and 80 females), out of which 101 were diabetics (with 59 on insulin, and 42 on oral hypoglycemics) with different duration of time in years, and 55 were non-diabetics. The data was analyzed after further subdividing the diabetics and non-diabetics into those having body mass index (BMI) $<25$, and those with $\mathrm{BMI} \geq 25$. 101 had $\mathrm{BMI} \geq 25$ (overweight/obese) [57 with $\mathrm{BMI}=25-29.9$ (overweight), and 44 with $\mathrm{BMI} \geq 30$ (obese)] and 55 had BMI= 18- 24.9 (normal weight). The individuals were then classified as those having insulin resistance syndrome (IRS) and those not having it. On the basis of NCEP criteria the associations between DM II, IRS, and obesity were evaluated. The credibility of three IR measuring parameters, namely, fasting insulin level (FIL), homeostatic model assessment (HOMA) and quick insulin sensitivity check index (QUICKI) was then assessed. Results: It was found that IRS prevailed up to $85 \%$ in diabetics and $76 \%$ in subjects with BMI $\geq 25$ out of which $84 \%$ IRS cases existed among obese (with BMI $\geq 30$ ). Similarly, $82 \%$ IRS cases were found diabetic and $72.4 \%$ with BMI $\geq 25$. FIL and HOMA showed significantly raised insulin and QUICKI showed significantly lower in IRS cases. But unlike FIL and HOMA, QUICKI did not show any significance when different subgroups of IRS cases were inter-compared. FIL and HOMA were also significantly raised in overweight/ obese individuals. QUICKI however showed significant but weak correlations with BMI. HOMA and QUICKI were significant in diabetics but FIL did not show significant difference. Conclusion: The results revealed strong correlation of obesity, IRS and DM II. HOMA was found better and sensitive index for evaluating IR in diabetes, IRS and obesity, than FIL alone or with QUICKI.
\end{abstract}

Keywords: Insulin Resistance Syndrome (IRS), Diabetes Mellitus Type II (DM II), Obesity, Body Mass Index (BMI), Fasting Insulin Level (FIL), Homeostatic Model Assessment (HOMA),

Quick Insulin Sensitivity Check Index (QUICKI)

\section{Introduction}

Diabetes mellitus type II (DM II) is the most rapidly growing life-long chronic degenerative metabolic disorder in the world, effecting mostly the adult life[1,2].In DM II, the two inter-related genetically determined pathological defects that occur many years before the development of disease, are $\beta$-cell dysfunction and insulin resistance (IR), which eventually lead to hyperglycemia [3,4]. The earliest detectable abnormality in DM II is probably the impairment of target tissues to respond to insulin, known as IR, necessitating extra insulin secretion in an attempt to stimulate the desensitized cells $[5,6,7,8]$.A variety of molecular and cellular defects have been implicated in 
the causation of IR. Obesity is considered as the single most important reversible causative environmental risk factor for IR, aggravating the metabolic disturbance in DM II[9].IR correlates with obesity in diabetics as well as in non-diabetics, developing gradually with weight gain [10,11].It is often quite marked in obese DM II cases and blood glucose control is often achieved at the expense of severe hyperinsulinemia [12].The central obesity however plays more significant role in IR than the peripheral obesity [13].The clustering of hyperinsulinemia with hyperglycemia, dyslipidemias, obesity (especially visceral adiposity), hypertension, coagulation abnormalities, and chronic subclinical inflammation is called insulin resistance syndrome (IRS) $[14,15]$. It is diagnosed clinically, by taking 5 variables: waist circumference, blood pressure, fasting blood glucose (FBG) level, high density lipoproteins (HDL) level and triglycerides (TG) level. The persons having disturbed levels in any three of these five criteria are classified as having IRS $[16,17]$.

FIL is a useful way to assess IR, but it alone has certain limitations, as for the same levels of fasting insulin, the hyperglycemia in diabetics would be much more pronounced than in non-diabetic insulin resistants [18].Similarly, the same non-elevated FIL may suggest either normal response to glucose or in DMII, an inadequate insulin secretion [19].HOMA and QUICKI taking into account FIL and BSL both, appear to behave as better and sensitive indicator for assessing IR rather than FIL alone, and as effective as the gold standard hyperinsulinemic euglycemic (HEC) technique [20,21]. Many researchers find QUICKI a better way to assess insulin sensitivity [18,22], whereas many consider it and HOMA both equally effective ways to assess the insulin sensitivity [23].Some found QUICKI to work best in nondiabetics [19], however contrastingly many researchers have found it to work equally effectively in patients of DM II also [20,21,22,23].

The aim of the study was to evaluate the association of obesity, IRS and DM II in Pakistani diaspora, and to explore the credibility of parameters of measures of IR namely FIL, HOMA and QUICKI in evaluating IR in obesity, IRS and DM II. These parameters may be used as diagnostic and prognostic markers for assessing IR (in diabetics, non-diabetics, obese, and non-obese) for assessing the degree of control this life style disease, so as to decrease the risk of future development of complications.

\section{Materials and Methods}

We conducted the study in Akhuwat Health Clinic Lahore, Pathology Laboratory Allama Iqbal Medical College Lahore, and Center for Research in Molecular Medicine University of Lahore, Pakistan.

\subsection{Subjects}

We included 156 subjects (76 males and 80 females) in the study, over 30 years of age, out of which 101 were diabetics (59 on insulin, and 42 on oral hypoglycemics) with different duration of time in years, and 55 were nondiabetics. We grouped the data by taking body mass index (BMI) of diabetics and non-diabetics into account, and further subdividing them into those having $\mathrm{BMI}<25$, and those with $\mathrm{BMI} \geq 25$. Out of 156,101 had $\mathrm{BMI} \geq 25$ (overweight/obese) [57 with $\mathrm{BMI}=25-29.9$ (overweight), and 44 with $\mathrm{BMI} \geq 30$ (obese)] and 55 had $\mathrm{BMI}=18-24.9$ (normal weight). Out of 101 diabetics, 66 were overweight/obese and 55 were normal weight, and out of 55 non-diabetics, 35 had $\mathrm{BMI} \geq 25$, and 20 with $\mathrm{BMI}<25$. We then evaluated the individuals clinically for IR on the basis of NCEP criteria for classifying IRS, and the associations between DM II, IRS, and obesity assessed. The IR in IRS, obesity, and DM II was then reassessed by measuring the three IR parameters: FIL, HOMA and QUICKI, and their credibility weighed.

\subsection{Body Mass Index (BMI)}

We recorded weight and height in $\mathrm{kg}$ and meters respectively, in light indoor clothes, without shoes, by weighing machine with affixed stadiometer. We calculated BMI by using the formula: weight in $\mathrm{kg} /$ height in $\mathrm{m}^{2}$. BMI below $25 \mathrm{~kg} / \mathrm{m}^{2}$ was taken as underweight, from 20$24.9 \mathrm{~kg} / \mathrm{m}^{2}$ as normal weight, from $25-29.9 \mathrm{~kg} / \mathrm{m}^{2}$ is as overweight $\&$ above $30 \mathrm{~kg} / \mathrm{m}^{2}$ as obese [24,25].

\subsection{Collection of Blood Sample}

For the estimation of fasting glucose, lipid profile and insulin levels, we took $5 \mathrm{ml}$ venous blood samples were drawn from ante-cubital vein using aseptic measures in $5 \mathrm{cc}$ disposable syringes without any anticoagulant in the morning after an overnight fast of 10-12 hours. Any type of medication, oral intake or smoking in the morning prior to the testing was not permitted. We allowed the blood to clot for half, after which we pipetted the supernatant clear fluid out soon into $10 \mathrm{cc}$ centrifuge tubes, with $10-100 \mu \mathrm{l}$ automated micropipette (Huwaii). These tubes were centrifuged at $3000 \mathrm{rpm}$ for 10 minutes. We then pipetted the clear serum in each tube was pipetted out into 2 clean dry Ependorff cups [2].We froze them at $-20^{\circ} \mathrm{C}$ to be used later on (for up to 30 days) for the estimation of blood lipids and insulin. We avoided repetitive freezing and thawing [26].We estimated blood sugar in each syringe/tube by using enzymatic kits by spectrophotometry.

\subsection{National Cholesterol Education Program (NCEP) 2001 Criteria for Evaluating IR}

We classified the individuals as IRS and non-IRS on the basis of clinically easily applicable parameters as described by NCEP (2001) definition for IR [16, 17]. If three or more of these factors were positive, we considered the case to be clinically insulin resistant:

- Waist circumference: $\geq 80 \mathrm{~cm}$ (in females), $\geq 90 \mathrm{~cm}$ (in males) 
- Blood pressure: $\geq 130 / 85 \mathrm{mmHg}$, or on antihypertensive treatment

- $\quad$ Fasting blood glucose (FBG): $\geq 110 \mathrm{mg} / \mathrm{dl}$, or on antidiabetic treatment

- $\quad$ Triglycerides (TGs): $\geq 150 \mathrm{mg} / \mathrm{dl}$, or on anti-lipaemic therapy

- High density lipoproteins (HDL): $\quad<50 \mathrm{mg} / \mathrm{dl}$ (in females), $<40 \mathrm{mg} / \mathrm{dl}$ (in males), or on anti-lipaemic therapy

\subsubsection{Waist Circumference}

We measured weight to the nearest $\mathrm{cm}$ in single indoor clothes by soft non-elastic tape midway between the lowest rib and the iliac crest, approximately over the level of umbilicus [24].

\subsubsection{Blood Pressure}

We recorded systolic and diastolic blood pressures in $\mathrm{mmHg}$, by Yamasu mercury sphygmomanometer, Japan and 3M Littmann classic II S.E. stethoscope, USA.

\subsubsection{Fasting Blood Glucose (FBG), and Triglycerides (TGs) Estimation}

We performed these biochemical analyses of serum by using enzymatic kits of Human TM, Germany. We pipetted $10 \mu \mathrm{l}$ of samples and standards, and $1000 \mu \mathrm{l}$ of reagent with Huawii automated micropipettes (10-50 $\mu 1,100-1000 \mu l)$. We mixed $10 \mu \mathrm{l}$ of standard and of each of the samples with $1000 \mu l$ reagent, in small test tube. The incubation was done in the water tub set at $37^{\circ} \mathrm{C}$, for 5 minutes. We then measured the absorbance of the samples and standard was against the reagent blank within 60 minutes, by automated spectrophotometer, Merck Microlab 300, Germany and recorded the values in $\mathrm{mg} / \mathrm{dl}$.

\subsubsection{High Density Lipoproteins (HDL) Estimation}

We estimated HDL by indirect method using enzymatic kit, Human TM, Germany. We pipetted $200 \mu$ l of sample or standard and $500 \mu$ l of reagent in $10 \mathrm{cc}$ centrifuge tubes, mixed, allowed to stand for 10 minutes at room temperature and then centrifuged at $4000 \mathrm{rpm}$ for 10 minutes. We then pipetted $100 \mu \mathrm{l}$ of this clear supernatant, and $1000 \mu \mathrm{l}$ of the cholesterol reagent into a small test tube, mixed, incubated again at $37^{\circ} \mathrm{C}$ for 5 minutes, and analyzed the results in the automated analyzer, against the reagent blank.

\subsection{Insulin Resistance Parameters}

\subsubsection{FIL (Fasting Insulin Level)}

We estimated insulin levels in the clear serum by enzyme linked immune-sorbent assay (ELISA), using 96 well Accubind Insulin Elisa Microwell kit, product code: 2425300, Monobind Inc USA. We used Star Fax-2200 Awareness Tech Inc incubator-shaker, Thermo Electron Corp well wash-aspirator, automated Humareader Single Plus AE microplate reader for incubating, shaking, wellwashing, aspirating and taking absorbance. We used manufacturer's reference range of $0.7-9.0 \mu \mathrm{U} / \mathrm{ml}$ for normal FIL.

\subsubsection{HOMA-IR (Homeostatic Model Assessment index for Insulin Resistance)}

We calculated HOMA by: Fasting Plasma Insulin (uU/ml) $x$ Fasting Plasma Glucose $(\mathrm{mM} / \mathrm{l}) / 22.5$. Referring to the cut-off values by different researchers, we took the arbitrary cut-off for HOMA- IR in the present study as 3.0, and considered the individuals above this cut-off value as having IR [24, 27, 28].

\subsubsection{QUICKI (Quantitative Insulin Sensitivity Check Index)}

We calculated QUICKI by: 1 / [log Fasting Plasma Insulin $(\mu \mathrm{U} / \mathrm{ml})+\log$ Fasting Plasma Glucose $(\mathrm{mg} / \mathrm{dl})]$. Referring to the general cut-off value by different researchers, we also took 0.3 as arbitrary cut off value, and considered the individuals below this cut-off value as having IR [19, 21].

\subsection{Statistical Analysis}

We performed all statistical analysis using SPSS software version 16, and set the levels of statistical significance at $\mathrm{P}<$ 0.05 . We calculated means \pm S.D., and variance of all the values in different groups. We did different comparisons and correlations by using independent sample t-test, ANOVA, Bonferroni's adjustment, Chi-square and Spearman correlation co-efficient.

\section{Results}

Out of 101 type II diabetics, $86(85.1 \%)$ had clinical IRS, while only $19(34.5 \%)$ amongst 55 non-diabetics had clinical IRS. The difference was significant with $\mathrm{P}<0.001$, showing increased incidence of IRS in DM II (Table 1).We found significant association of BMI with IRS $(\mathrm{P} \leq 0.005)$. The percentages of IRS cases increased and those of nonIRS cases decreased with increasing BMI. $52.7 \%$ of the subjects with BMI $<25$ had IRS, increasing to $68.4 \%$ of subjects with BMI 25-29.9 having IRS, further increasing to $84.1 \%$ with BMI $\geq 30$ having IRS cases. Similarly, the non-IRS cases decreased from $47.3 \%$ in subjects with $\mathrm{BMI}<25$, to $31.6 \%$ in those with BMI 25-29.9, finally to $15.9 \%$ in ones with $\mathrm{BMI} \geq 30$ (Table2).

Table 1. Distribution and comparison of IRS and non-IRS cases between diabetics and non-diabetics.

\begin{tabular}{|c|c|c|c|c|}
\hline & \multicolumn{2}{|c|}{ Diabetics } & \multicolumn{2}{|c|}{ Non-diabetics } \\
\hline & $\mathbf{N}$ & $\%$ & $\mathbf{N}$ & $\%$ \\
\hline Non Insulin Resistants & 15 & 14.9 & 36 & 65.5 \\
\hline With Insulin Resistant Syndrome & 86 & 85.1 & 19 & 34.5 \\
\hline
\end{tabular}


Table 2. Distribution of cases by BMI and IR.

\begin{tabular}{|c|c|c|c|c|c|}
\hline & & \multicolumn{2}{|c|}{ Non IRS } & \multicolumn{2}{|c|}{ IRS } \\
\hline & & 26 & 47.3 & 29 & 52.7 \\
\hline & & 25 & 23.75 & 76 & 76.25 \\
\hline a) & $25-29.9$ & 18 & 31.6 & 39 & 68.4 \\
\hline b) & $\geq 30$ & 7 & 15.9 & 37 & 84.1 \\
\hline Tot: & & 51 & & 105 & \\
\hline
\end{tabular}

Chi-square $=10.98 \quad \mathrm{P} \leq 0.005$

Table 3. Comparison of IR parameters between diabetics and non-diabetics.

\begin{tabular}{llllllllll}
\hline & \multicolumn{2}{l}{ Diabetics $(\mathbf{N}=\mathbf{1 0 1})$} & \multicolumn{4}{c}{ Non-diabetics $(\mathbf{N}=\mathbf{5 5})$} \\
& Mean & SD & Min & Max & Mean & SD & Min & Max & P-value \\
\hline FIL $(\mu \mathrm{U} / \mathrm{ml})$ & 13.3 & 14.1 & 0.1 & 87.6 & 10.5 & 8.23 & 0.1 & 32.5 & 0.110 \\
HOMA-IR & 5.22 & 5.31 & 0.03 & 32.4 & 2.09 & 1.65 & 0.02 & 7.14 & $<0.001$ \\
QUICKI & 0.34 & 0.1 & 0.24 & 0.93 & 0.38 & 0.13 & 0.29 & 1.15 & 0.023 \\
\hline
\end{tabular}

Table 4. Comparison of IR parameters between IRS and non-IRS.

\begin{tabular}{|c|c|c|c|c|c|c|c|c|c|}
\hline & \multicolumn{4}{|c|}{ IRS $(N=105)$} & \multicolumn{5}{|c|}{ Non-IRS $(\mathrm{N}=51)$} \\
\hline & Mean & SD & Min & Max & Mean & SD & Min & Max & P-value \\
\hline FIL $(\mu \mathrm{U} / \mathrm{ml})$ & 8.9 & 7.4 & 0.1 & 31.7 & 14.0 & 14.0 & 0.1 & 87.6 & 0.003 \\
\hline HOMA- IR & 2.2 & 1.7 & 0.0 & 6.3 & 5.1 & 5.3 & 0.1 & 32.4 & $<0.001$ \\
\hline QUICKI & 0.4 & 0.2 & 0.3 & 1.2 & 0.3 & 0.1 & 0.2 & 0.8 & 0.010 \\
\hline
\end{tabular}

Table 5. Distribution and comparison of IR parameters among 2 main groups of BMI.

\begin{tabular}{|c|c|c|c|c|c|c|c|c|c|}
\hline & \multicolumn{4}{|c|}{ BMI $<25(N=55)$} & \multicolumn{5}{|c|}{$\mathrm{BMI} \geq 25(\mathrm{~N}=101)$} \\
\hline & Mean & SD & Min & Max & Mean & SD & Min & Max & P-value \\
\hline FIL $(\mu \mathrm{U} / \mathrm{ml})$ & 8.8 & 7.6 & 0.1 & 32.0 & 14.2 & 14.1 & 0.1 & 87.6 & 0.002 \\
\hline HOMA IR & 2.9 & 2.4 & 0.0 & 12.0 & 4.8 & 5.4 & 0.0 & 32.4 & 0.003 \\
\hline QUICKI & 0.4 & 0.1 & 0.3 & 0.9 & 0.4 & 0.1 & 0.2 & 1.2 & 0.542 \\
\hline
\end{tabular}

Table 6. Comparison of different IR parameters between diabetic IRS and non-IRS cases .

\begin{tabular}{|c|c|c|c|c|c|c|c|c|c|}
\hline & \multicolumn{4}{|c|}{ Diabetic Non-IRS $(\mathrm{N}=15)$} & \multicolumn{4}{|c|}{ Diabetic IRS $(\mathbf{N}=86)$} & \multirow[b]{2}{*}{ P-value } \\
\hline & Mean & SD & Min & Max & Mean & SD & Min & Max & \\
\hline FIL $(\mu \mathrm{U} / \mathrm{ml})$ & 7.5 & 5.8 & 0.1 & 21.5 & 14.4 & 14.9 & 0.1 & 87.6 & 0.003 \\
\hline HOMA IR & 2.8 & 1.8 & 0.0 & 5.5 & 5.7 & 5.6 & 0.1 & 32.4 & $<0.001$ \\
\hline QUICKI & 0.4 & 0.2 & 0.3 & 0.9 & 0.3 & 0.1 & 0.2 & 0.8 & 0.190 \\
\hline
\end{tabular}

Table 7. Comparison of different IR parameters between IRS cases with and without diabetes.

\begin{tabular}{llllllllll}
\hline & \multicolumn{2}{l}{ IRS with Diabetes $(\mathbf{N}=\mathbf{8 6})$} & \multicolumn{4}{c}{ IRS without Diabetes $(\mathbf{N}=\mathbf{1 9})$} & \multicolumn{2}{c}{} \\
& Mean & SD & Min & Max & Mean & SD & Min & Max & P-value \\
\hline FIL $(\mu \mathrm{U} / \mathrm{ml})$ & 14.4 & 14.9 & 0.1 & 87.6 & 12.3 & 8.5 & 1.2 & 32.5 & 0.566 \\
HOMA IR & 5.6 & 5.6 & 0.1 & 32.4 & 2.4 & 1.7 & 0.2 & 7.1 & $<0.001$ \\
QUICKI & 0.3 & 0.1 & 0.2 & 0.8 & 0.4 & 0.0 & 0.3 & 0.5 & 0.205 \\
\hline
\end{tabular}

Table 8. Comparison of different IR parameters between IRS 'normal weight and overweight/obese' cases .

\begin{tabular}{llllllllll}
\hline & \multicolumn{2}{l}{ BMI $<\mathbf{2 5}(\mathbf{N}=\mathbf{2 9})$} & \multicolumn{3}{c}{ BMI $\geq 25$} & $(\mathbf{N}=\mathbf{7 6})$ & \multicolumn{3}{c}{} \\
& Mean & SD & Min & Max & Mean & SD & Min & Max & P-value \\
\hline FIL $(\mu \mathrm{U} / \mathrm{ml})$ & 8.6 & 7.1 & 0.1 & 32.0 & 16.1 & 15.4 & 0.4 & 87.6 & 0.001 \\
HOMA IR & 3.5 & 2.8 & 0.1 & 12.0 & 5.6 & 5.9 & 0.1 & 32.4 & 0.013 \\
QUICKI & 0.4 & 0.1 & 0.3 & 0.8 & 0.3 & 0.1 & 0.2 & 0.6 & 0.205 \\
\hline
\end{tabular}

Table 9. Comparison of different IR parameters among normal weight and overweight /obese groups in diabetic IRS cases.

\begin{tabular}{|c|c|c|c|c|c|c|c|c|c|}
\hline & \multicolumn{4}{|c|}{ BMI $>25(N=27)$} & \multicolumn{5}{|c|}{$B M I \geq 25(N=59)$} \\
\hline & Mean & SD & Min & Max & Mean & SD & Min & Max & P-value \\
\hline FIL $(\mu \mathrm{U} / \mathrm{ml})$ & 8.4 & 7.3 & 0.1 & 32.0 & 17.1 & 16.7 & 0.4 & 87.6 & 0.001 \\
\hline HOMA IR & 3.6 & 2.8 & 0.1 & 12.0 & 6.6 & 6.3 & 0.1 & 32.4 & 0.003 \\
\hline QUICKI & 0.4 & 0.1 & 0.3 & 0.8 & 0.3 & 0.1 & 0.2 & 0.6 & 0.108 \\
\hline
\end{tabular}


Amongst IRS cases, there were more cases of BMI $\geq 25$ $(72.4 \%)$ compared to $27.6 \%$ cases with $\mathrm{BMI}<25$. Amongst non-IRS cases, there are likewise comparatively lesser subjects with $\mathrm{BMI} \geq 25(49 \%)$ than those with $\mathrm{BMI}<25$ $(51 \%)$.

The strong association of BMI with IR was also shown by FIL and HOMA. It was observed that those having BMI $\geq 25$ had mean FIL significantly higher $(14.2 \pm 14.1 \mu \mathrm{U} / \mathrm{ml})$ than those with BMI $<25(8.8 \pm 7.6 \mu \mathrm{U} / \mathrm{ml})$, with $\mathrm{P} \leq 0.002$. Similarly, HOMA-IR values were also significantly higher in people with $\mathrm{BMI} \geq 25(4.8 \pm 5.4)$ than those with $\mathrm{BMI}<$ 25 (2.9 \pm 2.4$)$, with $\mathrm{P} \leq 0.003$. The comparison made by ANOVA b/w 3 BMI groups tells that those having BMI $<25$, had average FIL of $8.8 \pm 7.6 \mu \mathrm{U} / \mathrm{ml}$, those with BMI between 25--29.9 had $11.9 \pm 10.8 \mu \mathrm{U} / \mathrm{ml}$, while those with $\mathrm{BMI} \geq 30$ had $17.2 \pm 17.1 \mu \mathrm{U} / \mathrm{ml} \quad(\mathrm{P} \leq 0.003)$. In pair-wise (Bonferonni's) comparisons, those with BMI $\geq 30$ had mean FIL difference of $8.37 \mu \mathrm{U} / \mathrm{ml}$ with $\mathrm{BMI}<25$ (P = 0.002). Similarly, the mean difference between overweight $(\mathrm{BMI}=25--29.9)$ and obese $(\mathrm{BMI} \geq 30)$ was $5.28 \mu \mathrm{U} / \mathrm{ml}$, with $\mathrm{P}=0.092$, which may be considered clinically significant. Although the overweight group (BMI = 25-29.9) had higher mean FIL $(11.9 \mu \mathrm{U} / \mathrm{ml})$ than normal weight group $(8.8 \mu \mathrm{U} / \mathrm{ml})$ yet, the difference wasn't significant. Similarly the differences in HOMA-IR by ANOVA, were significant $(\mathrm{P} \leq 0.001)$. The obese had highest average HOMA-IR $=6.26 \pm 6.59$, the over-weights had $3.64 \pm 3.88$ and the normal weights had the lowest $=2.89 \pm 2.40$. Pairwise comparisons tell us that obese group had significantly higher HOMA-IR than normal $(\mathrm{P} \leq 0.001)$ and over-weight groups $(\mathrm{P} \leq 0.01)$, by the differences of 3.37 and 2.62, respectively. Although HOMA-IR values for over-weights were higher (3.64) than normal weights (2.89), yet the difference wasn't significant. The differences in QUICKI b/w 3 groups of BMI weren't significant (tables not included) (Table 5).

Three measures were used for observing IR in diabetics. Although mean FIL was higher in type II diabetics (13.3 \pm $14.1 \mu \mathrm{U} / \mathrm{ml})$ than in non-diabetics $(10.5 \pm 8.23 \mu \mathrm{U} / \mathrm{ml})$, but the difference in FIL was insignificant $(\mathrm{p}=0.110)$, may be due to the possible presence of IRS in non-diabetics, too. HOMA-IR and QUICKI however gave significant differences between type II diabetics $(5.2 \pm 5.31,0.34 \pm 0.1)$ and non-diabetics $(2.09 \pm 1.65,0.38 \pm 0.13)$ with $\mathrm{P} \leq$ 0.001 and 0.02 respectively. HOMA-IR was significantly higher and QUICKI significantly lower in type II diabetics than in non-diabetics (Table 3).The differences in insulin resistance parameters i.e. FIL, HOMA-IR, and QUICKI among IRS and non-IRS cases were significant, with $\mathrm{P}=$ $0.003,<0.001$ and 0.010 , respectively. The FIL and HOMA-IR were significantly higher and QUICKI lower in IRS cases $(14 \pm 14 \mu \mathrm{U} / \mathrm{ml}, 5.1 \pm 5.3$ and $0.3 \pm 0.1)$ than in non- IRS ones $(8.9 \pm 7.4 \mu \mathrm{U} / \mathrm{ml}, 2.2 \pm 1.7,0.4 \pm 0.2)$ (Table4). The differences in insulin resistance parameters i.e. FIL and HOMA-IR among diabetic IRS and diabetic non-IRS cases were likewise found significant, with $\mathrm{P}<$
0.005 and $<0.001$, respectively. The FIL and HOMA-IR were significantly higher in diabetic IRS cases $(14 \pm 14.9$ $\mu \mathrm{U} / \mathrm{ml}$ and 5.7 \pm 5.6$)$ than in non- IRS ones $(7.5 \pm 5.8 \mu \mathrm{U} / \mathrm{ml}$, $2.8 \pm 1.8,0.4 \pm 0.2)$. Although QUICKI values in diabetic IRS cases were less $(0.3 \pm 0.1)$ than diabetic non-IRS cases $(0.4 \pm 0.2)$, yet the difference was not significant (Table6).

Out of 105 IRS cases, 86 were diabetic and 19 nondiabetic. The only significant difference observed for IR parameters between diabetic IRS cases and non-diabetic IRS cases was found for mean HOMA-IR, with $\mathrm{P}<0.001$, diabetics having mean HOMA value $=5.6 \pm 5.6$, while nondiabetics had values $=2.4 \pm 1.7$. The difference in mean FIL, although higher in diabetic IRS cases $(14.4 \pm 14.9 \mu \mathrm{U} / \mathrm{ml})$ than non-diabetic IRS cases $(12.3 \pm 8.5 \mu \mathrm{U} / \mathrm{ml})$, was insignificant. Similarly the difference in QUICKI values, although lower in diabetic IRS (0.3) than non-diabetic IRS cases (0.4), was insignificant $(\mathrm{P}=0.205)$ (Table 7). Amongst 105 IRS cases, 29 had BMI $<25$ and 76 with BMI $\geq 25$. Fasting Insulin levels almost doubled in the later group $(16.1 \pm 15.4 \mu \mathrm{U} / \mathrm{ml})$ from the former one $(8.6 \pm 7.1 \mu \mathrm{U} / \mathrm{ml})$, with $\mathrm{P} \leq 0.001$. Mean HOMA-IR values were also significantly higher in group $2(5.9 \pm 0.1)$ than group 1 (3.5 \pm 2.8$)$, with $\mathrm{P} \leq 0.01$. QUICKI values although lower in subjects with $\mathrm{BMI} \geq 25(0.3)$ than those with $\mathrm{BMI}<25$ (0.4), yet the difference was insignificant (Table 8).

Now, out of 86 diabetic IRS cases, 59 had BMI $\geq 25$ and 27 had BMI $<25$. The mean fasting insulin levels were significantly higher in those with BMI $\geq 25$ $(17.1 \pm 16.7 \mu \mathrm{U} / \mathrm{ml})$ than those with BMI $<25$ $(8.4 \pm 7.3 \mu \mathrm{U} / \mathrm{ml})$, with $\mathrm{P} \leq 0.001$. HOMA-IR was also significantly higher in preceding group $(6.6 \pm 6.3)$ than the later group (3.6 \pm 2.8$)$, with $\mathrm{P}=0.003$. The difference in mean QUICKI values, although lower in diabetic IRS subjects with $\mathrm{BMI} \geq 25(0.3)$ than those with BMI <25 (0.4), yet was statistically insignificant $(\mathrm{P}=0.108)$.

\section{Discussion}

Insulin resistance is a key component of and a common finding in type II diabetes, as a consequence of obesity, attributed to life style modifications (over-eating with sedentary life). IR is considered to be the main underlying cause in $70-80 \%$ type II diabetics. Development of IR being a slow and gradual process, begins with excess weight gain, years before the occurrence of overt diabetes. About $80 \%$ of the type II diabetics are obese. Obesity aggravates metabolic disturbance along with insulin resistance $[9,14]$.In the present study, an attempt was made to determine the association of IR with DM II (its major outcome) and obesity (its main causative factor) in Pakistani diaspora.

The study showed significant number of IRS cases in diabetics $(85.1 \%)$ than in non-diabetics $(34.5 \%)$. Only $15 \%$ diabetics did not have IRS. Some studies showed that over $74 \%$ of NIDDM patients were insulin resistant, almost 
correlating with the ratio of obesity in diabetes $(>80 \%)$, and about $18 \%$ of general population was estimated to have IR [16]. Others found 90\% [29], 78\% [13], 58\% [30], and 46\% [31] IRS cases in diabetic population.

Increased hepatic production of glucose and increased resistance to the actions of insulin leading to decreased peripheral utilization of glucose are the characteristic features of the disease [32]IR is the earliest detectable abnormality in DM II [8] and one of the most important triggers to the development of DM II [33].

The study showed the significant association of BMI with the occurrence of IRS. The percentages of IRS cases increased while those of non-IRS cases decreased with the increasing BMI. $52.7 \%$ of the subjects with $\mathrm{BMI}<25$ had IRS, increasing to $68.4 \%$ IRS cases in those having BMI between $25-29.9$, further increasing to $84.1 \%$ IRS cases in ones with BMI $\geq 30$. Similarly, the non-IRS cases decreased from $47.3 \%$ in subjects with $\mathrm{BMI}<25$, to $31.6 \%$ in those with BMI 25--29.9, finally to $15.9 \%$ in ones with BMI $\geq 30$. Out of 105 total IRS cases in the data, $72.4 \%$ had BMI $\geq 25$ compared to $27.6 \%$ with $\mathrm{BMI}<25$. Out of total of 51 non-IRS cases in the data, there are $49 \%$ subjects with BMI $\geq 25$ compared to $51 \%$ with $\mathrm{BMI}<25(51 \%)(\mathrm{P}=0.007)$. So, the findings correlate with those of the other researchers, where IR is shown to correlate with obesity in diabetics as well as non-diabetics [10, 11]. The chances of IR and metabolic disturbance are much increased if DM II patients are obese [12, 34].Increased occurrence of IRS is reported in obese DM II patients than non-obese ones [35], and that of raised FIL in obesity [18]. More mean HOMA values were found for obese IR cases than non-obese IR cases [36]

We used three measures of IR i.e. FIL, HOMA, and QUICKI for evaluating it. We found all three to be significant parameters of IR in IRS, whereas HOMA and QUICKI in diabetes, and FIL and HOMA in overweight/obese cases. Although mean FIL was higher in type II diabetics than in non-diabetics, but the difference in FIL was insignificant. It might be due to insulin sensitizing therapies, the presence of IRS cases in non-diabetics and the dwindling levels of insulin in the later stages of disease in DM II patients. So, HOMA-IR and QUICKI which take into account FIL and BSL both proved to be more efficient determinants of IR in diabetics rather than FIL alone. When different comparisons were made to analyze the associations of diabetes and obesity among IRS cases (tables 6, 7, 8, 9), QUICKI did not show any significant difference. FIL once again did not show significant difference when diabetic IRS cases were compared with non-diabetic IRS ones (table 7). Whereas all comparisons showed significantly raised HOMA values. In short, HOMA was found preferable to FIL and QUICKI if one were to evaluate IR. Researchers have found HOMA and QUICKI to behave as better and sensitive indicator for assessing IR rather than FIL alone, and as effective as the gold standard hyperinsulinemic euglycemic (HEC) technique [20, 29]. Many researchers find QUICKI a better way to assess insulin sensitivity $[18,20]$, whereas many consider it and HOMA both equally effective ways to assess the insulin sensitivity [23]. Some found QUICKI to work best in non-diabetics [19], however contrastingly many researchers have found it to work equally effectively in patients of DM II also [20,21, 22, 23].

\section{Conclusion}

The prevalence of IRS is more in obese DMII than nonobese ones. IRS was observed up to $85 \%$ in diabetics, $84 \%$ in obese and $76 \%$ in overall $\mathrm{BMI} \geq 25$. Vice versa, $82 \%$ IRS cases were found diabetic, and $72.4 \%$ overweight/obese. If one were to evaluate IR, HOMA was found preferable to FIL alone and QUICKI, as its measure.

\section{Acknowledgements}

We are thankful to Akhuwaat Health Clinic Lahore, Umer Sani Welfare Clinic Raiwind, Diabetic Clinic Jinnah Hospital Lahore, CRIMM Laboratory University of Lahore and Clinical Pathology Laboratory Allama Iqbal Medical College Lahore for facilitating the data collection and the conductance of the experimental work. Our special gratitude for Dr. Tahir Rasool, Mr. Bilal, Sister Maria, Dr Rohina Baloch and Mr Asim Jajja for their valuable support and cooperation in the accomplishment of this project.

\section{References}

[1] T. Santoso, "Prevention of cardiovascular disease in diabetes mellitus: by stressing the CARD study," Acta Med Indones, vol. 32, pp.97-102, 2006.

[2] M. Verma ,S. Paneri, P. Badi, and P. G. Raman, “ Effect of increasing duration of diabetes mellitus type 2 on glycated hemoglobin and insulin sensitivity,'Ind J Clin Biochem, vol. 21, pp.142-146, January 2006.

[3] S. Inchiostro, "Measurements of insulin sensitivity in type-2 diabetes mellitus: Comparison between KITT and HOMA indices and evaluation of their relationship with the components of the insulin resistance syndrome," Diabetes UK Diabetic Med, vol. 22, pp. 39-44, 2004.

[4] G.M. Reaven, and B.B. Hoffman, "A role for insulin in the etiology and course of hypertension," Lancet, vol. 2, pp.435-437, 1987.

[5] B. Kim, "Blood sugar and insulin: The essentials,"2005. Retrieved from: http://drbenkim.com/articles-blood sugar .html, on $9^{\text {th }}$ March 2010.

[6] S. Schrinner, W.A. Scherbaum S.R. Bornstein, and Al Barthe, "Molecular mechanism of insulin resistance," Diabetes UK Diabetic Med, vol.22, pp.674-682, 2005.

[7] M. Laakso,'Insulin resistance and its impact on the approach to therapy of type-2 diabetes mellitus," Int J Clin Pract Suppl, vol.121, pp.8-12, 2001.

[8] R. A. Defronzo, R. C. Bonadonna, and E. Ferrannini, "Pathogenesis of NIDDM: A balanced overview," Diabetes Care, vol. 15, pp.318-368, March 1992. 
[9] M. B. Davidson, "Clinical implications of insulin resistance syndrome," Am J Med, vol.99, pp.420-426, 1995.

[10] O. E. Odeleye, M. De Courten, D. J. Pettitt, and E. Ravussin, "Fasting hyperinsulinemia is a predictor of increased body weight gain and obesity in Pima Indian children," Diabetes, vol. 46, pp.1341-1345, 1997.

[11] E. J. Boyko, D. L. Leonetti, R. W. Bergstrom, L. M. Newell, and W.Y. Fujimoto, "Low insulin secretion and high fasting insulin and C-peptide levels predict increased visceral adiposity,” Diabetes, vol. 45, pp.1010-1015, 1996.

[12] N. Ali, N. Afzal, S. Shahjahan, M. Z. Ahmed, and A. Baseer, "A study of insulin resistance in obese and non-obese healthy FDRs of T2DMpatients," Pak Postgrad Med J, vol. 15, pp.81-92, February 2004.

[13] N. Shah, Amanullah, and S. Ataullah, "Prevalence of insulin resistance in type-2 diabetic patients," J Postgrad Med Inst, vol. 22, pp. 52-56, January 2008.

[14] N. Ali, N. Afzal, M. Z. Ahmed, S. Shahjahan, and A. S. Sheikh, "Obesity indices and lipid levels in healthy relatives of type 2 diabetes mellitus patients," Pak Postgrad Med J, vol. 14, 189-193, April 2003.

[15] P. Arner. Insulin resistance in type 2 diabetes: Role of fatty acids. Diab Metab Res Rev.2002; 18: S5-S9.

[16] C. E. Tam, S. Ma, D. Wai, S. K. Chen, and E. S. Tai, "Can we apply the National Cholesterol Education Program Adult Treatment Panel definition of the metabolic syndrome to Asians?" Diabetes Care, vol. 27, pp.1182-1186, May 2004.

[17] NCEP, "Third Report of NCEP expert panel on detection, evaluation and treatment of high blood cholesterol in adults (Adult treatment Panel III)," JAMA, vol. 285, pp.486-497, September 2001.

[18] J. D. Bagdade, E. L. Bierman, and D. Porte, "The significance of basal insulin levels in the evaluation of the insulin response to glucose in diabetic and non-diabetic subjects," J Clin Inves, vol. 46, pp.1549-1557, October 1967.

[19] R. Mack, B. Skurnick, Y. S. Jean, M. P. Nobre, and D. Bigg, "Fasting insulin levels as a measure of insulin resistance in American Blacks," J Appl Res, vol. 4, pp.90-94, January 2004.

[20] V. D. Castracane, and R. P. Kauffman, "Controlling PCOS, Part 1: Assessing insulin sensitivity," Contemporary OG/GYN, January 2003.

[21] K.A. McAuley, S. M. Williams, J. I. Mann, R. J. Walker, N, J. L. Barned, L. A. Temple, and A. W. Duncan, "Diagnosing insulin resistance in the general population," Diabetes Care, vol. 24 , pp.460-464, 2001.

[22] A. Katz, S. S. Nambi, K. Mather, A. D. Baron, D. A. Follmann, G. Sullivan, and M. J. Quon, "Quantitative insulin sensitivity check index: a simple accurate method for assessing insulin sensitivity in humans," J Clin Endocrinol Metab, vol. 85, pp.2402-2410, July 2007.

[23] H. Yokoyama, M. Emoto, S. Fujiwara, K. Motoyama, T. Morioka, M. Komatsu et al, " Quantitative insulin sensitivity check index and the reciprocal index of homeostasis model assessment are useful indexes of insulin resistance in type 2 diabetic patients with wide range of fasting glucose" J Clin Endocrinol Metab,vol. 89, pp.14811484,March 2004.

[24] S. Katoh, M. Lehtovirta, J .Kaprio, V. Harjutsalo, M. Kosekenvuo, and J. Eriksson, "Genetic and environmental effects on fasting and post-challenge plasma glucose and serum insulin values in Finnish twins," J Clin Endocrinol Metab, vol.90, 2642-2647, 2005.

[25] G. A. Bray,and R. F. H. Hamman, "Insulin resistance and pre-diabetes", 2008. Retrieved from: http://diabetes.niddk.nih.gov/DM/pubs/insulinresistanceon $9^{\text {th }}$ March 2010

[26] W. Hseuh, and A. Collins, Eds. Plasma Insulin (Linco ELISA): In Animal Models of Diabetic Complications Consortium Protocols, Version 1, December 2004 Dec.

[27] D. R. Mathews, J. P. Hosker, A. S. Rudenski, B. A. Naylor, D. F. Treacher,and R. C. Turner, " Homeostasis Model Assessment: Insulin resistance and $\beta$-cell function from fasting plasma glucose and insulin concentrations in man”,Diabetologia, vol.28, pp.412-419, 1985.

[28] S. Schoppen, B. Cano, I. D. Oya, L. L. Simon, P. Riestra ,and M. D. Oya, "Relationship between leptin, insulin level and homeostatic model assessment (HOMA) index in Spanish pubertal children, "Summer Meeting, Proceedings of the Nutrition Society,"67(OCE8), E381, June $30^{\text {th }}$ - July $3^{\text {rd }} 2008$.

[29] E. Bonora, S. Kiechl, J. Williet, F. Oberhollenzer, G. Egger, G. Targher, et al. Prevalence of insulin resistance in metabolic disorders: The Bruneck Study. Diabetes, vol. 47, pp.1643-1649, 1998.

[30] N. Ali, N. Afzal, J. Subazwari, A. Ahmed, A. Abbas, and A. Sultan, "Insulin resistance syndrome in type 2 diabetes mellitus patients,'Esculapia J Serv Inst Med Sci, vol. 3, pp.20-22, January 2007.

[31] S. M.S. Ashraf, F. Ziauddin, and U. Jehangir, "Metabolic syndrome in type-2 diabetes mellitus," Pak J Med Sci, vol. 22, pp.295-299, 2006.

[32] A. C. Guyton, and J. E. Hall, "Insulin, glucagon and diabetes mellitus," Textbook of Medical Physiology, $11^{\text {th }}$ ed. Philadelphia, PA: Elsevier, 2009; pp.974-975.

[33] D. R. Mathews, "Insulin resistance and $\beta$-cell dysfunction: A clinical perspective,"Diab, Obes and Metab, vol. 3, pp. 2833, January 2001

[34] F. Masud, M. Hassan, S. Abaidullah, and I. Alam,"Assessment of metabolic profile and body mass index (BMI) in type II diabetics treated with Metformin and insulin,” Pak J Med Sci, vol.9,pp.29-34. January 1991.

[35] H. Maegawa H, A. Kashiwagi, and Y. Shigeta, "Obesity as a risk factor for developing non-insulin dependent diabetes mellitus, obesity, and insulin, insulin resistance," Nippon Naibumpi Gakkai Zasshi.1995; vol. 71, pp.97-104, February 1995.

[36] M. Keskin, S. Kurtoglu, M. Kendirci, M. E. Atabek, and C. Yazisi, "Homeostasis model assessment is more reliable than the fasting glucose / insulin ratio and quantitative insulin sensitivity check index for assessing insulin resistance among obese children and adolescents," Pediatrics, vol.115, pp. 500-503, 2005 